\title{
Effective pseudo-potentials of hydrodynamic origin
}

\author{
By TODD M. SQUIRES \\ Department of Physics, Harvard University, Cambridge, MA 02138
}

(Received 7 February 2001)

\begin{abstract}
It is shown that low Reynolds number fluid flows can cause suspended particles to respond as though they were in an equilibrium system with an effective potential. This general result follows naturally from the fact that different methods of moving particles in viscous fluids give rise to very different long-range flows. Two examples are discussed: electrophoretic 'levitation' of a heavy charged sphere, for which a hydrodynamic 'pseudo-potential' can be written in closed form, and quasi-two dimensional crystals of like-charged colloidal spheres which form near charged walls, whose apparent attraction arises not from a force but from persistent fluid flows.
\end{abstract}

\section{Introduction}

It is well-known that a particle which is moving under the influence of an external force such as gravity in a viscous fluid sets up a disturbance flow in the fluid which, in the low Reynolds number limit, decays with distance like $r^{-1}$. The long-range character of these force-driven flows manifests itself in many situations. One example involves collective diffusive behavior. A pair of Brownian spheres has been predicted (Batchelor 1976) and measured (Crocker 1997) to diffuse in a correlated manner. On the basis of these long-range correlations, Crocker et al. (2000) have developed a new technique for the microrheology of complex materials. Recently, Dufresne et al. (2000) experimentally and theoretically showed the diffusive behavior of a pair of Brownian spheres to be strongly influenced even by distant solid boundaries. Long-range flows also have a significant influence on the properties of sedimenting systems, giving rise to numerous divergent integrals (see e.g. Hinch 1988). For example, Caflisch \& Luke (1985) have predicted velocity fluctuations in sedimenting particulate suspensions to diverge with system size, and Brenner (1999) has drawn attention to the fact that sedimenting particles' long-range interaction with cell walls can screen the flows and cut off the divergent fluctuations. In addition, long-range hydrodynamic coupling has provided a plausible interpretation (Squires \& Brenner 2000) for the experiment of Larsen \& Grier (1997), which measured an apparent attraction between like-charged colloidal spheres in the proximity of a similarlycharged wall.

It is also well-known that there are other ways to move particles which do not give rise to such long-range disturbance velocity fields. One example is electrophoresis (e.g. Anderson 1989), wherein charged particles move under the influence of an applied electric field. A charged particle in solution attracts oppositely charged counter-ions from the solution, which form a screening 'double-layer' (e.g. Russel et al. 1989, chap. 4). The particle/double layer system is electrically neutral, so an applied electric field exerts no net force on the ensemble. However, the charged particle and the counter-ions are driven 
in opposite directions, giving an apparent 'slip velocity' at the edge of the double layer, and a net motion to the particle. Since velocity fields with $r^{-1}$ decay arise only from a net force on the fluid, electrophoretic (and all force-free) flow fields must decay at least as fast as $r^{-2}$.

In this paper we will demonstrate that this difference between the long-range disturbance flows due to forced and force-free motions can be exploited to cause particles to behave as though they were subject to equilibrium forces derived from an effective potential. As an example, we will discuss the electrophoretic 'levitation' of a heavy charged sphere off a wall, for which an effective pseudo-potential $\Phi_{\text {ps }}$ can be determined exactly. We will then present a method for making like-charged colloidal spheres near a wall behave as though there was an apparent attraction between them, so that layered like-charged colloidal crystals can be grown. Throughout this work, we use the term 'pseudo-potential' to emphasize that the hydrodynamic component of $\Phi_{\mathrm{ps}}$ arises not from a force, but from persistent low-Reynolds number flows which entrain each particle.

We shall first briefly review the hydrodynamics of forced- and force-free motion. Colloidal particles $(\sim 1 \mu \mathrm{m})$ and their typical velocities $(\sim 1 \mu \mathrm{m} / \mathrm{s})$ are small enough that inertial effects are entirely negligible. In this limit, a rigid sphere of radius $a$ moves due to an external force $\mathbf{F}$ at velocity $\mathbf{U}_{\mathbf{0}}=b_{0} \mathbf{F}$, where $b_{0}=(6 \pi \eta a)^{-1}$ is the Stokes mobility. This motion sets up a disturbance flow in the surrounding fluid

$$
\mathbf{u}(\mathbf{R})=\frac{1}{8 \pi \eta}\left(\frac{\mathbf{I}}{R}+\frac{\mathbf{R R}}{R^{3}}\right) \cdot \mathbf{F}-\frac{a^{2}}{24 \pi \eta}\left(-\frac{\mathbf{I}}{R^{3}}+\frac{3 \mathbf{R R}}{R^{5}}\right) \cdot \mathbf{F},
$$

where $\eta$ is the fluid viscosity and $\mathbf{R}=\mathbf{r}-\mathbf{r}_{0}$ is the vector connecting the observation point $\mathbf{r}$ and the center of the sphere $\mathbf{r}_{0}$. This can be re-expressed using fundamental singularities of the Stokes equation as

$$
\mathbf{u}(\mathbf{r})=\frac{1}{8 \pi \eta}\left\{\mathbf{S}(\mathbf{R})-\frac{a^{2}}{3} \nabla \nabla \cdot\left(\frac{1}{R}\right)\right\} \cdot \mathbf{F},
$$

The first term represents the flow due to a point force, known as a Stokeslet, which decays like $R^{-1}$. The second term is the Green's function for a point source dipole, also called a potential dipole, which decays like $R^{-3}$. The flow far from a forced sphere is dominated by the Stokeslet, which depends on neither the size nor the velocity of the sphere.

By contrast, a charged insulating sphere with a thin double-layer moving due to a uniform electric field $\mathbf{E}_{\infty}$ sets up a disturbance flow outside the double layer (e.g. Russel et al. 1989 , p. 256),

$$
\mathbf{u}_{E}(\mathbf{R})=\frac{\epsilon \zeta}{4 \pi \eta} \frac{a^{3}}{2}\left(-\frac{\mathbf{I}}{R^{3}}+\frac{3 \mathbf{R R}}{R^{5}}\right) \cdot \mathbf{E}_{\infty},
$$

where $\epsilon$ is the dielectric constant of the fluid and $\zeta$ is the potential difference across the double-layer. Here the sphere moves at velocity $\mathbf{V}_{\mathbf{0}}=M_{0} \mathbf{E}_{\infty}$, where $M_{0}=\epsilon \zeta / 4 \pi \eta$ is the electrophoretic mobility. This flow is exclusively potential dipole flow and decays like $R^{-3}$.

Walls affect these flows significantly, since the fluid velocity must vanish identically on solid boundaries. Image singularities, analogous to image charges in electrostatics, can often be found which exactly cancel a flow set up on the wall. In this paper, we shall make extensive use of the flow due to a Stokeslet (point force) $F \hat{\mathbf{z}}$ oriented perpendicular to a wall, which Blake (1971) found to be

$$
\mathbf{u}(\mathbf{R})=\frac{F}{8 \pi \eta}\left(\mathbf{S}(\mathbf{R})-\mathbf{S}\left(\mathbf{R}^{i}\right)+\left.2 h^{2}\left\{\nabla_{0} \nabla_{0}\left(\frac{1}{r}\right)\right\}\right|_{\mathbf{R}^{i}}-\left.2 h\left\{\frac{\partial \mathbf{S}(\mathbf{r})}{\partial z_{0}}\right\}\right|_{\mathbf{R}^{i}}\right) \cdot \hat{\mathbf{z}}
$$


where $\mathbf{R}^{i}=\mathbf{R}+2 h \hat{\mathbf{z}}$ is the vector between the images and the observation point, derivatives with respect to the source point $\mathbf{r}_{0}$ (denoted by $\nabla_{0}$ ) should be taken first, and then the position $\mathbf{R}^{i}$ inserted in place of $\mathbf{r}$. The flow due to the images decays like $R^{-1}$, whereas the image flow for a source dipole (Blake \& Chwang 1974), which will not be required in this paper, decays like $R^{-3}$.

The velocity of a sphere forced toward a wall has two contributions: it moves at $\mathbf{U}_{\mathbf{0}}$ due to the applied force, and it is advected with the flow set up by the image singularities. Evaluating the image flow from (1.4) at $r_{0}$ results in an $O(a / h)$ wall correction to the (scalar) mobility for motion perpendicular to the wall (e.g. Happel \& Brenner 1983):

$$
b_{\perp}(h)=b_{0}\left(1-\frac{9 a}{8 h}+O\left[\frac{a^{3}}{h^{3}}\right]\right) .
$$

The correction to the electrophoretic mobility can be derived in similar fashion, but is slightly more complicated because the wall gives both hydrodynamic and electrostatic contributions. Since the image flow (and electrostatic image potential) for electrophoretic motion decays like $R^{-3}$, the wall correction to the electrophoretic mobility is $O\left(a^{3} / h^{3}\right)$, and has been calculated using the method of reflections by Keh \& Anderson (1985) to give

$$
M_{\perp}(h)=M_{0}\left(1-\frac{5 a^{3}}{8 h^{3}}+O\left[\frac{a^{5}}{h^{5}}\right]\right) .
$$

Throughout this paper, we will treat $a / h$ and $a / r$ as small, and so neglect this $O\left(a^{3} / h^{3}\right)$ variation in $M_{\perp}$.

\section{Electrophoretic 'levitation'}

These two motions and their respective flows can be superposed, since Stokes' flow is linear. A charged sphere which sediments due to a force $F_{\mathrm{g}}$ towards a planar electrode and which is electrophoretically driven upwards due to a uniform electric field $\mathbf{E}_{\infty}$ will have a velocity $v$ given by

$$
v=-b_{\perp}(h) F_{\mathrm{g}}+M_{\perp}(h) E_{\infty},
$$

with $b_{\perp}(h)$ and $M_{\perp}(h)$ given by (1.5) and (1.6) respectively. If the bulk electrophoretic velocity is less than the bulk sedimentation velocity, so that $\psi=M_{0} E_{\infty} / b_{0} F_{\mathrm{g}}<1$, there will be a unique height

$$
\frac{h_{0}}{a}=\frac{9}{8(1-\psi)}+O\left(a^{2}\right)
$$

where the two velocities exactly balance and a non-Brownian sphere will come to rest. A sphere started above $h_{0}$ falls faster than electrophoresis drives it up, and it moves down towards the plane. Similarly, a sphere below $h_{0}$ falls too slowly, and electrophoresis drives it up. Thus $h_{0}$ is a stable steady location. On the other hand, $h_{0}$ would be an unstable steady point for a sphere falling away from a wall.

A sphere undergoing Brownian motion will have a probability distribution $P(h)$ of being located at $h$ which is given by the steady Fokker-Planck equation (see e.g. Batchelor 1977)

$$
\frac{\partial P}{\partial t}=0=-\nabla \cdot \mathbf{J} \equiv-\nabla \cdot\left[\mathbf{v}(h) P-k_{B} T \mathbf{b}(h) \cdot \nabla P\right],
$$

where $k_{\mathrm{B}} T \mathbf{b}$ is a tensor diffusivity. This equation has solution $P(h)=P_{0} \exp \left(-\Phi_{\mathrm{ps}} / k_{B} T\right)$, 
where $\Phi_{\mathrm{ps}}$ is a non-equilibrium 'pseudo-potential'

$$
\Phi_{\mathrm{ps}}(h)=F_{\mathrm{g}} h-\int^{h} \frac{M_{\perp}\left(h^{\prime}\right) E_{\infty}}{b_{\perp}\left(h^{\prime}\right)} d h^{\prime} .
$$

Inserting the values of $b_{\perp}$ and $M_{\perp}$ from (1.5) and (1.6) and keeping terms to $O(a / h)$, we integrate (2.4) to obtain

$$
\Phi_{\mathrm{ps}}(h) \approx F_{\mathrm{g}} h-\frac{M_{0} E_{\infty}}{b_{0}}\left[h+\frac{9 a}{8} \ln \left(\frac{h}{a}-\frac{9}{8}\right)\right],
$$

which can be expanded about its minimum at $h_{0}$ to give an approximately harmonic well

$$
\frac{\Phi_{\mathrm{ps}}}{k_{B} T} \sim \frac{8}{9} \frac{F_{\mathrm{g}} a}{k_{B} T}\left[\frac{(1-\psi)^{2}}{\psi}\right] \frac{\left(h-h_{0}\right)^{2}}{2 a^{2}}+\frac{\Phi_{\mathrm{ps}}\left(h_{0}\right)}{k_{B} T} .
$$

If one viewed only the motion of a single sphere in this configuration, one would not be able to distinguish between this non-equilibrium pseudo-potential and a true thermodynamic potential. However, the physical distinction is crucial: the only force on the sphere/double layer system is $F_{\mathrm{g}}$, directed toward the wall.

\section{Multi-particle pseudo-potential interactions}

Even though the electrophoretically 'levitated' particle is stationary, it drives a longrange flow. Far from the sphere, the flow is always dominated by the force-driven motion, since it decays the most slowly. Streamlines for the flow around a hovering sphere, described in section 2, are shown in figure 1.

A second identical sphere, placed at the same height and at some distance $r$ away from the original sphere, will both set up a long-range flow as well as be entrained by the flow set up by the first sphere. Forcing two spheres perpendicular to the wall induces a relative velocity between them (Squires \& Brenner 2000), in the plane of the wall, as is evident from the streamlines in figure 1 . This relative velocity of sphere 1 arises from its entrainment in the flow set up by the images of sphere 2, and is found to $O(a / h)$ and $O(a / r)$ from (1.4) to be

$$
\dot{x}_{1}=-\dot{x}_{2}=-\frac{3}{2 \pi \eta} \frac{r h^{3}}{\left(r^{2}+4 h^{2}\right)^{5 / 2}} F_{g},
$$

where $x$ is the coordinate parallel to the wall, along the line joining the spheres, as in figure 2. Two spheres forced away from a wall move together as they leave the wall, whereas two spheres falling towards a wall move apart. In this case, we observe that the relative motion between two non-Brownian hovering spheres resembles the motion due to an effective repulsive force, even though it does not arise from a force. Example trajectories for two such spheres are shown by the solid curves in figure 2, with arrows showing the collapse onto these trajectories from various initial configurations.

\section{Like-charge colloidal crystal formation}

Finally, we explore the possibility of setting up an attractive interaction between the spheres, which can lead to crystal formation. Since forcing two spheres toward a wall led to an effective repulsion, forcing them off of the wall will give an effective attraction. One way to do this would be to electrophoretically drive neutrally buoyant charged spheres into a similarly-charged wall. This would require the wall to possess a static surface charge density and to simultaneously passes a current. 


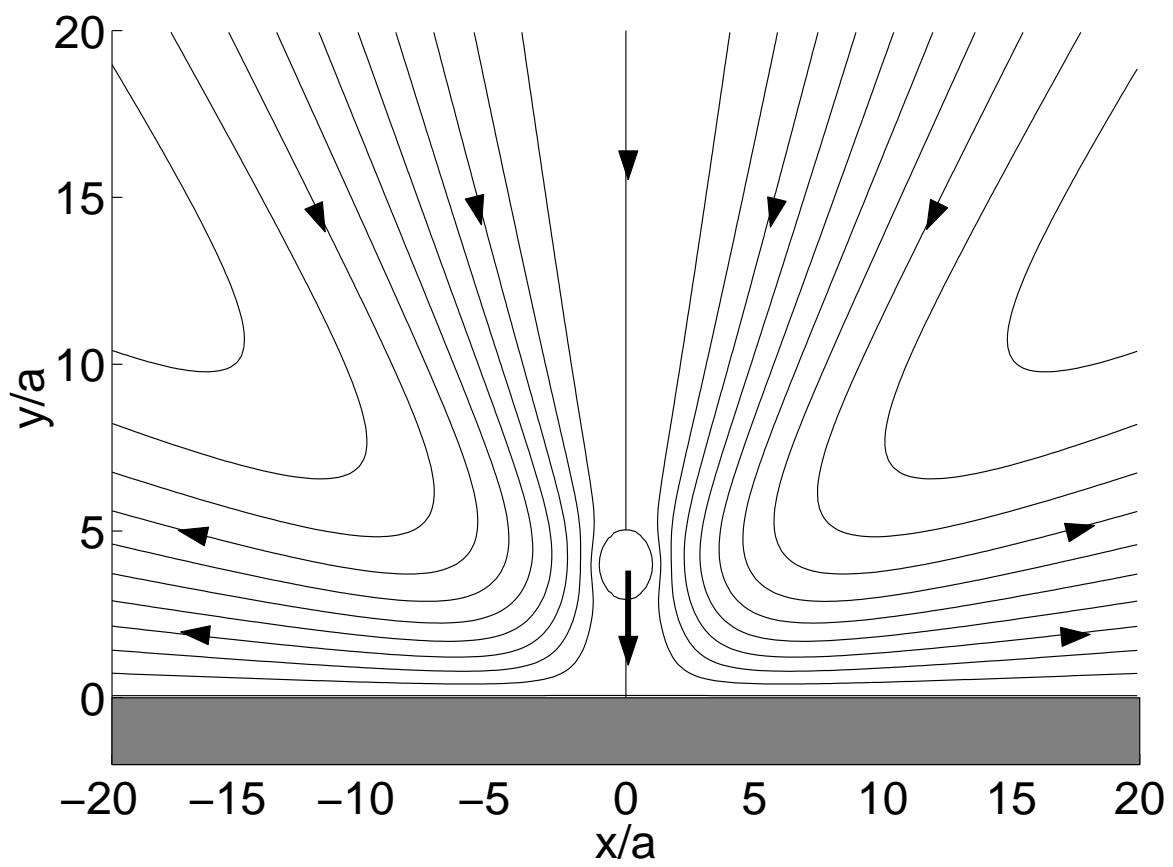

FIgURE 1. Streamlines for a heavy charged sphere electrophoretically levitated at $h_{0}=4 a$. Flows move down and radially out along the streamlines. The closed streamline shows the slip velocity on the sphere surface. The flow decays slowly and asymptotically approaches the flow due to a falling sphere near a wall, even though the sphere is immobile. Gravity exerts a force directed toward the wall, and given a sphere with positive $\zeta$ potential, the electric field is directed away from the wall.

As we have seen, the far-field hydrodynamic flows are dominated by the net force on the body and its charge cloud. In this case, the net force $F_{\mathrm{w}}$ on each is given by its electrostatic repulsion from the static charge on the wall, found by integrating an electrostatic stress tensor over the surface of the sphere (see, e.g. Russel et al. 1989). Under the linear superposition approximation, the repulsive force the wall exerts on the sphere is found to be

$$
\frac{F_{\mathrm{w}}}{k_{\mathrm{B}} T}=4 \pi Z \sigma_{\mathrm{g}} \lambda_{\mathrm{B}} \frac{e^{\kappa a}}{1+\kappa a} e^{-\kappa h},
$$

where $Z$ is the effective charge on the sphere, $\sigma_{\mathrm{g}}$ is the charge density on the wall, $\kappa^{-1}$ is the Debye screening length and $\lambda_{\mathrm{B}}$ is the Bjerrum length $(\approx .7 \mathrm{~nm}$ in water at room temperature). This force sets up a flow around each sphere like in figure 1, with flow arrows reversed. The lateral component of the flow now draws the spheres together.

Two non-Brownian spheres separated by distance $r$ are immobilized at the height $h_{0}$ where $\mathbf{M} \cdot \mathbf{E}_{\infty}=\mathbf{b}\left(h_{0}, r\right) \cdot \mathbf{F}_{\mathrm{w}}\left(h_{0}\right)$. The equivalent force on each sphere which would be necessary to drive this motion in a quiescent fluid is

$$
F_{x_{1}} \approx \frac{\dot{x_{1}}}{b_{x_{1} x_{1}}-b_{x_{1} x_{2}}},
$$

where $b_{x_{1} x_{2}}$ is the coefficient of $\mathbf{b}$ which gives the mobility sphere 1 in the $\hat{\mathbf{x}}$ direction due to a force on sphere 1 in the same direction, and so on. Using (3.1), and neglecting 


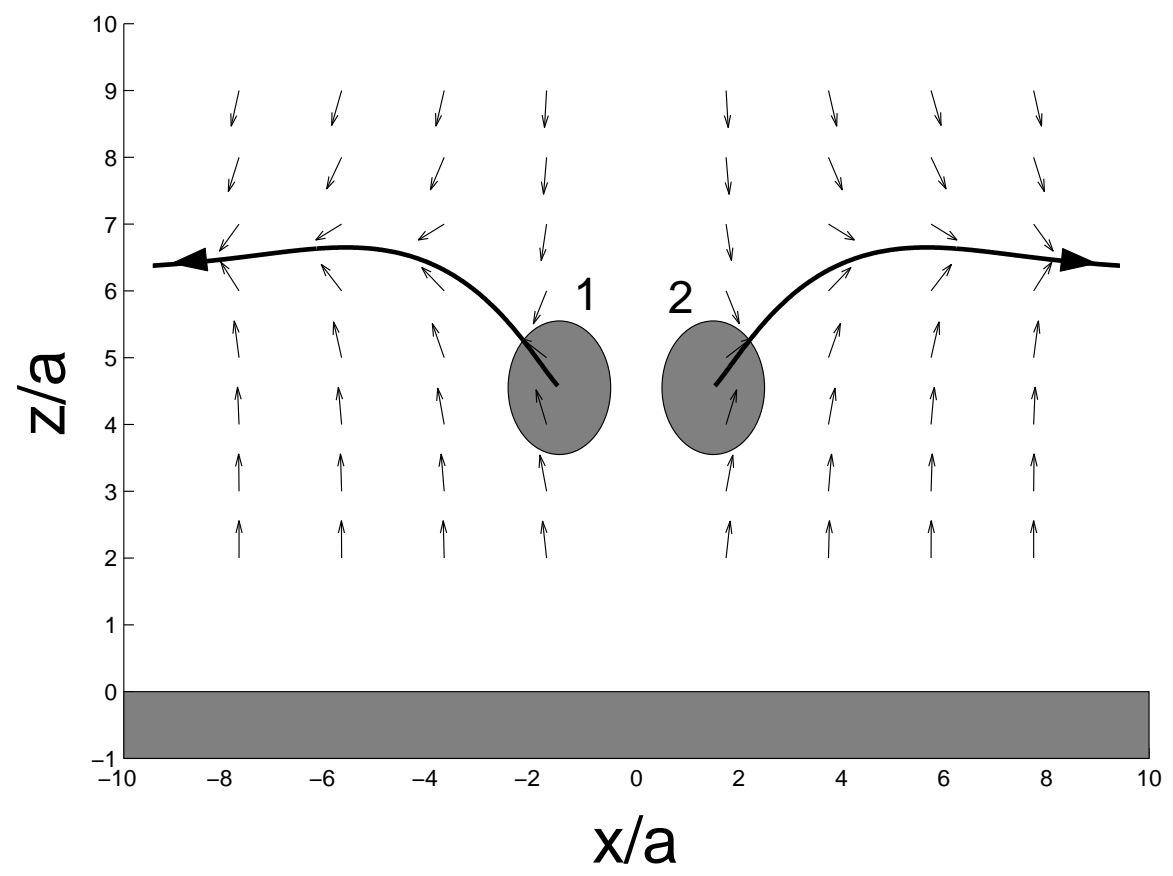

FIGURE 2. Two heavy spheres electrophoretically driven upwards approach a stable trajectory, on which the spheres move apart. This mimics a repulsion between the spheres, and asymptotes to two spheres levitating independently. In this figure, we have chosen $E$ such that an independent sphere would hover at $h=6 a$.

the weak dependence of $h_{0}$ upon $r$, this can be integrated to yield a pseudo-potential

$$
\Phi_{\mathrm{ps}}\left(r, h_{0}\right) \approx U_{\mathrm{p}}(r)-\frac{3 h_{0}^{3} F_{\mathrm{w}}\left(h_{0}\right)}{2 \pi \eta} \int^{r} \frac{r\left(4 h_{0}^{2}+r^{2}\right)^{-5 / 2}}{b_{x_{1} x_{1}}\left(h_{0}\right)-b_{x_{1} x_{2}}\left(r, h_{0}\right)} d r,
$$

where $U_{\mathrm{p}}$ is the true pair potential between the spheres. Although $b_{x_{2} x_{1}}$ is $O(a)$, in practice it is small compared to $b_{x_{1} x_{1}}$, and its neglect allows the direct integration of (4.3) to give

$$
\Phi_{\mathrm{ps}}\left(r, h_{0}\right) \approx U_{\mathrm{p}}(r)-\frac{F_{\mathrm{w}}\left(h_{0}\right)}{1-\frac{9 a}{16 h_{0}}} \frac{3 h_{0}^{3} a}{\left(4 h_{0}^{2}+r^{2}\right)^{3 / 2}} .
$$

The true pair potential between charged spheres, $U_{\mathrm{p}}$, is given in linear superposition approximation by (e.g. Larsen \& Grier 1997),

$$
\frac{U_{\mathrm{p}}}{k_{B} T}=Z^{2} \lambda_{B}\left(\frac{e^{\kappa a}}{1+\kappa a}\right)^{2} \frac{e^{-\kappa r}}{r} .
$$

Equation (4.4) is identical to that derived by Squires \& Brenner (2000) in their analysis of the experiment of Larsen \& Grier (1997) which measured the effective interaction between like-charged colloidal spheres near a single charged wall. Although the two formulae were derived in the same fashion, they have very different physical consequences. In that experiment, a pair of spheres was repeatedly released from the same height $h_{0}$ and then re-trapped; therefore, a net force systematically drove the spheres away from the wall throughout the experiment. Consequently, there was a systematic relative motion of the spheres towards each other which had been interpreted to arise from an attractive force. In an equilbrium system, where the spheres fluctuate into the wall as often as away, 
one would expect no systematic relative motion, and this hydrodynamic effect would be of no physical consequence.

Similarly, the pseudo-potential of equation (4.4) does not describe an attractive force between two like-charged colloids. However, in the present situation, similarly-charged spheres actually do respond as though there was an attraction. The effect of electrophoresis is to 'pin' the spheres at some height without applying a force, so that the long-range flow is dominated by the forced motion and entrains neighboring spheres accordingly. A collection of spheres can thus aggregate to form clusters-a behavior which might otherwise seem to arise from an attractive force.

Spheres which are not neutrally buoyant have a similar pseudo-potential, with $F_{\mathrm{w}}-F_{\mathrm{g}}$ in place of $F_{\mathrm{w}}$ in (4.4). Note that both the depth and the range of this potential can be tuned independently-the range by adjusting the electric field $E_{\infty}$ to give different heights $h_{0}$ and the overall magnitude of the attraction by adjusting the relative density of the particle to affect $F_{\mathrm{g}}$. Furthermore, one could study the freezing and melting of the crystals we'll describe next by simply adjusting $E_{\infty}$ during an experiment.

Brownian motion considerably complicates the above picture. Again, the probability distribution for the spheres' positions obey the steady Fokker-Planck equation (2.3). An interesting fact is that there is a persistent non-zero probability current $\mathbf{J}$ in the steady state. The probability current can only be zero when

$$
-\nabla \Phi_{\mathrm{ps}}=\mathbf{b}^{-1} \cdot \mathbf{u}
$$

has a solution. In equilibrium systems, $\mathbf{u}=\mathbf{b} \cdot \mathbf{F}$ so that $\mathbf{F}=-\nabla \Phi_{\mathrm{ps}}$ as expected. Here $\mathbf{u}=\mathbf{b} \cdot \mathbf{F}+\mathbf{u}_{E}$, and (4.6) will not in general have a solution. Physically, this is because the equivalent force required to give velocity $\mathbf{u}_{E}$ would be non-conservative. Vertical fluctuations take the pair into regions of stronger or weaker attraction, leading to persistent closed orbits of $\mathbf{J}$. When these vertical fluctuations are relatively small, $\mathbf{J}$ is small and the effective pseudo-potential is well approximated by (4.4). A comparison betweeen (4.4) and simulated results is shown in figure 3 . The lower two pseudo-potential wells (a) and (b) are deep enough that bound states form. The simulated pseudo-potentials $\Phi_{\mathrm{ps}} / k_{B} T$ are thus simply - $\log g(r)$, where $g(r)$ is the the pair correlation function obtained from simulations of a pair of spheres. We used the method described by Crocker \& Grier (1996) to simulate the shallowest potential well (c), since it is not deep enough for bound states.

We have run simulations which account for Brownian motion to demonstrate that spheres can indeed self-assemble into a quasi-2D crystal. We use the dynamical equations

$$
\mathbf{v}=\mathbf{b} \cdot \mathbf{F}+\mathbf{M} \cdot \mathbf{E}
$$

where $\mathbf{v}, \mathbf{b}$ and so on are multiparticle quantities. We retain terms up to $O(a)$ in $\mathbf{b}$ and $\mathbf{M}$ and use a box of side $100 a$ with periodic boundaries. Brownian motion is accounted for in standard fashion (Ermak \& McCammon 1978; Grassia et al. 1995), wherein $\mathbf{D}=$ $k_{B} T \mathbf{b}$ is a position-dependent tensor diffusivity. We use as input parameters the values $Z=7300, \kappa^{-1}=.275 \mu m$ and $2 a=.652 \mu m$ as in the experiments of Larsen \& Grier (1997) and the value $\sigma_{\mathrm{g}}=.4 \sigma_{\mathrm{s}}$ for the (unmeasured) charge density on the wall which Squires \& Brenner (2000) found as the best fit to the experimental data. We choose these values because 1) the effects we are discussing are most pronounced with long screening lengths and high surface charge densities, so that the spheres tend to 'hover' at an appreciable distance from the wall, and 2) these effects may bear some relation to these particular experiments. We started nineteen spheres in a hexagonal crystal, then simulated their dynamics until it was clear that the qualitative features would not change. This was typically approximately a minute or less of real time. Figure 3 shows the resulting suspensions at the end of each run for three different field strengths. The 
strongest field, for which $h_{0}=2 \mu m$, yields a hexagonal crystal, whereas a weaker field, for which $h_{0}=2.2 \mu \mathrm{m}$, is strong enough to yield a dense phase, but not strong enough to retain orientational order. We loosely term this an istropic 'liquid' phase. Finally, for an even weaker field, for which $h_{0}=2.5 \mu \mathrm{m}$, the attraction is sufficiently weak that the spheres spread out to fill the box as an isotropic 'gas'.

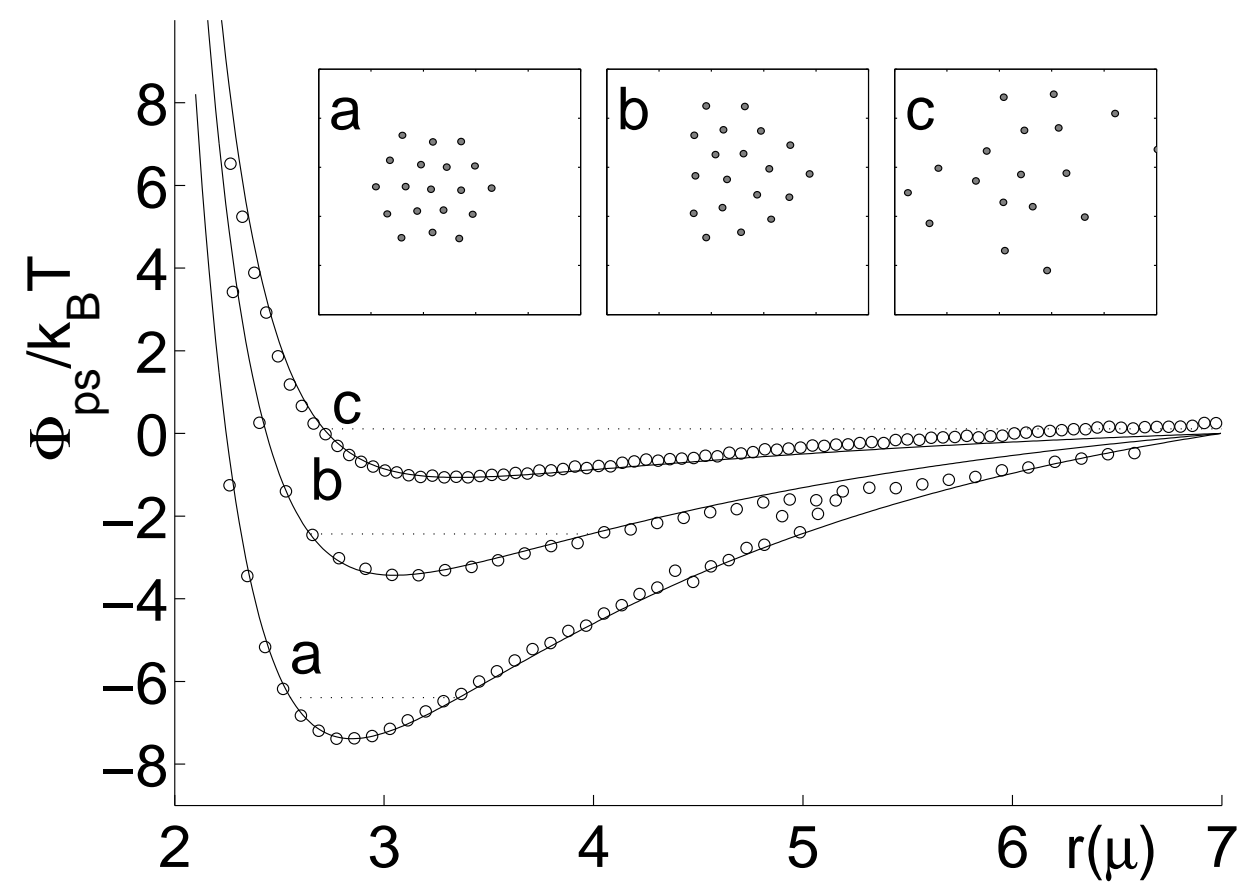

FiguRE 3. Radial pseudo-potentials and representative 'phases' of neutrally buoyant charged spheres electrophoretically driven into the double-layer surrounding a charged wall. a) A strong field $\left(h_{0}=2 \mu \mathrm{m}\right)$ yields a deep pseudo-potential well and tight pairwise confinement, giving a regular triangular lattice. b) A moderate field $\left(h_{0}=2.2 \mu m\right)$ yields a pseudo-potential well deep enough to yield a dense phase, but not strong enough to retain orientational order. c) A weak field $\left(h_{0}=2.5 \mu \mathrm{m}\right)$ yields an isotropic 'gas'. Dotted lines represent $1 k_{B} T$ of energy and give typical excursions about the equilibrium point. Lines give the approximate analytic form (4.4), and dots give the simulated pseudo-potentials.

\section{Discussion and conclusions}

While we have specifically investigated the combination of electrophoretically-driven motion and body-force motion, the present results have a wider validity. Our results will hold whenever motion using body forces such as gravity, electrostatic repulsion or attraction, magnetic forces, etc. are combined with force-free motion. There are many ways to achieve the latter. Since any self-propelled motion can not result in a net force or torque on the system, all swimmers generate flows decaying faster than $r^{-1}$ (Lighthill 1976). There are also other types of "phoretic" motion, which involve particle motion due to fields which interact with the particle's surface (e.g. Anderson 1989), such as thermophoresis (motion due to thermal gradients) and diffusophoresis (motion due to solute concentration gradients). Similarly, the thermocapillary motion of bubbles (Young et al. 1959), driven by thermal surface tension gradients, is force-free.

Having shown that like-charged colloidal crystals can self-assemble into ordered crystals 
due to long-range persistent non-equilibrium hydrodynamic flows, it is natural to ask whether there could be any connection between these crystals and those observed by Larsen \& Grier (1997). These metastable crystallites formed near the charged glass walls of the experimental cells, and persisted for times orders of magnitude longer than purely repulsive Brownian spheres should. This response can certainly be understood within the present picture, and would require a force-free motion to drive the spheres into the charge cloud of the walls. A spurious current in the cell could do this; however, it is unlikely that this explains Larsen and Grier's crystals, for two reasons: 1) there are no electrodes on the top and bottom walls of their cells, and 2) the crystals were observed to form on both the top and bottom walls, whereas a net current in the cell would likely drive the spheres to one wall or the other. Another possible current source, suggested by D. S. Fisher, is the dissociation of ionic groups on the glass surface. Other possible mechanisms for force-free motion include thermal or electrolyte concentration gradients; however, it is not clear which if any of these mechanisms are present or responsible for the crystals. It is generally believed that a novel long-ranged attraction between confined like-charged colloids, whose origin remains controversial, gives rise to this behavior (e.g. Hansen \& Lowen 2000). The present work provides a non-equilibrium mechanism whose origins are well-known and understood which would mimic an attractive interaction and could drive like-charge colloidal crystallization, so its potential role should be investigated further.

Two-dimensional colloidal crystals have been formed on electrodes via electrophoretic deposition (Bohmer 1996; Trau et al. 1997), and Solomentsev et al. (1997) has proposed a electrohydrodynamic model, which bears some similarity to the present work, for its explanation. The crucial difference between the our work and that of Solomentsev et al. (1997) and is that in the latter, the particles are first deposited on the electrode, and then electro-osmotic flows drive adjacent particles towards one another. These electro-osmotic flows are much shorter-ranged than the body-force driven flows described here, and are highly screened by the close proximity of the wall.

In conclusion, we have used the difference in flow fields set up by force-driven motion and force-free motion to show that persistent long-range viscous flowfields can be set up, resulting in particle motion which mimics an effective potential. This appears surprising at first, since the system is out of equilibrium, and yet behaves as though it is an equilibrium system with an effective potential which is hydrodynamically driven. Given the general nature of this effect, these persistent flowfields are likely to find various applications in self-assembly and microfluidic contexts.

This work was supported under the NSF Division of Mathematical Sciences grant DMS-9733030.

\section{REFERENCES}

Anderson, J. L. 1989 Colloid transport by interfacial forces. Ann. Rev. Fluid Mech. 21, 61-99.

BAtchelor, G. K. 1976 Brownian diffusion of particles with hydrodynamic interaction. J. Fluid Mech. 74, 1-29.

Batchelor, G. K. 1977 Developments in microhydrodynamics. In Theoretical and Applied Mechanics (ed. W. T. Koiter), pp. 33-55. North Holland Publishing.

Blake, J. R. 1971 A note on the image system for a stokeslet in a no-slip boundary. Proc. Camb. Phil. Soc. 70, 303-310.

Blake, J. R. \& Chwang, A. T. 1974 Fundamental singularities of viscous flow 1. image systems in the vicinity of a stationary no-slip boundary. J. Eng. Math 8, 23-29.

Bohmer, M. 1996 In situ observation of 2-dimensional clustering during electrophoretic depositoin. Langmuir 12, 5747-5750. 
Brenner, M. P. 1999 Screening mechanisms in sedimentation. Phys. Fluids 11, 754-772.

CAflisch, R. E. \& Luke, J. H. C. 1985 Variance in the sedimentation speed of a suspension. Phys. Fluids 28, 759-760.

Crocker, J. C. 1997 Measurement of the hydrodynamic corrections to the brownian motion of two colloidal spheres. J. Chem. Phys 106, 2837-2840.

Crocker, J. C. \& Grier, D. G. 1996 Methods of digital video microscopy for colloidal studies. J. Colloid Interface Sci. 179, 298-310.

Crocker, J. C., Valentine, M. T., Weeks, E. R., Gisler, T., Kaplan, P. D., Yodh, A. G. \& Weitz, D. A. 2000 Two-point microrheology of inhomogeneous soft materials. Phys. Rev. Lett. 85, 888-891.

Dufresne, E. R., Squires, T. M., Brenner, M. P. \& Grier, D. G. 2000 Hydrodynamic coupling of two brownian spheres to a planar surface. Phys. Rev. Lett. 85, 3317-3320.

Ermak, D. L. \& McCammon, J. A. 1978 Brownian dynamics with hydrodynamic interactions. J Chem. Phys. 69, 1352-1360.

Grassia, P. S., Hinch, E. J. \& Nitsche, L. C. 1995 Computer simulations of brownian motion of complex systems. J. Fluid. Mech. 282, 373-403.

HAnsen, J. P. \& Lowen, H. 2000 Effective interactions between electric double layers. Ann. Rev. Phys. Chem. 51, 209-242.

Happel, J. \& Brenner, H. 1983 Low Reynolds Number Hydrodynamics. The Hague: Martinus Nijhoff Publishers.

Hinch, E. J. 1988 Sedimentation of small particles. In Disorder and Mixing (ed. E. Guyon, J.-P. Nadal \& Y. Pomeau), pp. 153-161. Kluwer Academic Publishers.

KeH, H. J. \& Anderson, J. L. 1985 Boundary effects on electrophoretic motion of colloidal spheres. J. Fluid Mech. 153, 417-439.

Larsen, A. E. \& Grier, D. G. 1997 Like-charge attractions in metastable colloidal crystallites. Nature 385, 230-233.

Lighthill, J. 1976 Flagellar hydrodynamics. SIAM Review 18, 161-230.

Russel, W. B., Saville, D. A. \& Schowalter, W. R. 1989 Colloidal Dispersions. Cambridge: Cambridge University Press.

Solomentsev, Y., Bohmer, M. \& Anderson, J. L. 1997 Particle clustering and pattern formation during electrophoretic depositions: a hydrodynamic model. Langmuir 13, 60586068.

Squires, T. M. \& Brenner, M. P. 2000 Like-charge attraction and hydrodynamic interaction. Phys. Rev. Lett 85, 4976-4979.

Trau, M., Saville, D. A. \& Aksay, I. A. 1997 Assembly of colloidal crystals at electrode interfaces. Langmuir 13, 6375-6381.

Young, N. O., Goldstein, J. S. \& Block, M. J. 1959 The motion of bubbles in a vertical temperature gradient. J. Fluid Mech. 6, 350-356. 